\title{
EL MERCADO INTERNACIONAL DE TEJIDOS DE ALGODÓN EN 1913 Y LA INDUSTRIA ESPAÑOLA
}

\author{
ANNA CARRERAS MARÍN \\ Universidad de Barcelona
}

\section{RESUMEN}

La escasa orientación exportadora de la industria algodonera española ha sido objeto de un amplio debate en la historia económica. Las aportaciones de los distintos autores a este debate se han centrado fundamentalmente en análisis de las características propias de la economía española. Mi propuesta consiste en enfocar el debate desde la perspectiva de las nuevas teorías del comercio internacional. Bajo este enfoque teórico, se plantea la existencia de competencia imperfecta en los mercados de tejidos de algodón en el período anterior a la Primera Guerra Mundial. Los modelos de competencia imperfecta pretenden ponderar econométricamente variables tales como la dependencia histórica, las influencias geográficas, el mercado interior y las estrategias de diferenciación de producto y segmentación de los mercados. La adopción de un enfoque comparativo a escala internacional permite sugerir algunas claves interpretativas para explicar la escasa relevancia exportadora de la industria algodonera española.

\section{ABSTRACT}

There are many studies about the export failure of the Spanish cotton industry, but they are mainly focused on specific features of the Spanish economy. This article applies new trade theories to this topic. Taking into account this theoretical framework, the main hypothesis is the imperfect competition of international cotton textile markets in 1913. The econometric model employed in this paper measures the influence of variables such as historical and geographical dependencies, home market effect and product differentiation strategies. This international approach shows new explanations to the lack of an export orientation of the Spanish cotton industry.

\section{INTRODUCCIÓN}

El estudio que se presenta aborda, desde un enfoque centrado en las llamadas nuevas teorías del comercio internacional, una cuestión amplia- 
mente debatida en la historia económica española: el fracaso exportador de la industria algodonera. Para ello, el análisis se enmarca en el ámbito internacional y se lleva a cabo una comparación entre los principales Estados exportadores de tejidos de algodón en el período anterior a la Primera Guerra Mundial, a partir de datos correspondientes al año 1913. Los supuestos teóricos de partida consisten en la presunción de imperfección de los mercados internacionales, debida a una compleja amalgama de variables de naturaleza tan distinta como las dependencias históricas derivadas de pasados coloniales, los condicionantes geográficos, la influencia de la demanda interna o la presencia de estrategias de diferenciación de producto.

La metodología seguida se basa en la aplicación de los modelos gravitacionales. Cabe señalar que esta opción metodológica presenta algunas debilidades en cuanto a su soporte teórico, al mismo tiempo que resulta parcial, pues no contempla los costes de los factores como variables explicativas. Esta parcialidad explicativa de los modelos gravitacionales puede compensarse mediante los llamados modelos Heckscher-Ohlin ampliados, basados en la teoría de las dotaciones de factores y que incorporan algunas de las variables geográficas, políticas y de demanda postuladas por las nuevas teorías del comercio internacional. La aplicación de este tipo de modelos Heckscher-Ohlin ampliados se contempla como un objetivo posterior de la investigación que ahora se presenta, en la que los modelos gravitacionales son sólo una primera aproximación.

Las conclusiones fundamentales de este tipo de análisis deben valorarse considerando sus dos limitaciones más importantes: la no inclusión de las variables factoriales en el modelo y la necesidad de una mayor profundización en el análisis cualitativo acerca de la naturaleza de los mercados de tejidos de algodón. Los resultados obtenidos destacan la relevancia de la hipótesis de imperfección de los mercados internacionales a partir de tres fenómenos distintos: la presencia de procesos de histéresis histórica en los antiguos mercados coloniales, la especificidad de los flujos de comercio intraindustrial en los intercambios intraeuropeos y la posibilidad de estrategias de competitividad alternativas a los precios, es decir, la calidad y la diferenciación de producto.

En el primer apartado se exponen las características y limitaciones de la fuente principal utilizada en la construcción de la base de datos. En el siguiente se plantean las hipótesis acerca de la naturaleza de los mercados de tejidos de algodón en el año 1913. El tercer apartado consiste en la especificación de la ecuación gravitacional, cuya estimación se presenta 
en el apartado siguiente. Por último, se concluye con la síntesis de los resultados y se plantean las cuestiones que habría que abordar en desarrollos posteriores de la investigación.

\section{LA FUENTE PRINCIPAL DE LA BASE DE DATOS: ESTADÍSTICAS ALEMANAS PARA LA INDUSTRIA TEXTIL MUNDIAL}

La base de datos utilizada se basa, principalmente, en la explotación de una fuente poco conocida en la literatura del sector textil pero con un gran potencial para el desarrollo de comparaciones internacionales. Se trata de la obra de A. Kertesz, Die Textilindustrie sämtlicher Staaten. Entwicklung, Erzeugung, Absatzverhältnisse. Nach den statistischen Unterlagen der einzelnen Staaten für die Baumwoll-, Woll-, Seiden-, Leine-, Juteund Konfektions-Industrie als zweite Auflage der «Textilindustrie Deutschlands im Weltbandel» ${ }^{1}$. Consiste en un compendio de estadísticas sobre la producción, el consumo, la exportación y la importación de materias primas así como de tejidos textiles, correspondientes a 133 Estados en 1913, incluyendo algunos enclaves territoriales que funcionaban como puertos francos.

Los datos se presentan con distintos niveles de desagregación. Geográficamente, la información aparece de forma individualizada para cada uno de los Estados, siguiendo una clasificación basada en su localización por continentes. Para alguno de ellos, también se aporta información a escala regional, aunque esta información no aparece de forma generalizada ni homogénea. Por sectores la información se presenta, primero, de forma agregada para toda la industria textil y, a continuación, de forma separada para la industria algodonera, la de la lana, la de la seda, la del lino, la del yute y la de la confección. Según el proceso de elaboración, la información aparece diferenciada para materias primas, tejidos semi-elaborados, tejidos acabados y confecciones. A diferencia del resto, esta información no es exhaustiva, ya que sólo recoge los principales datos para cada tipo de producto. En cuanto a las macromagnitudes económicas, se aportan datos sobre la producción, el consumo, las exportaciones y las importaciones para cada Estado y para cada sector textil.

\footnotetext{
${ }^{1}$ Kertesz (1917), La industria textil mundial. Evolución, producción y ventas. Según las estadisticas estatales referentes a la industria algodonera, la de la lana, la de la seda, la del lino, la del yute y la de la confección, segunda edición de "La industria textil alemana en el comercio mundials.
} 
Entre las características más relevantes de esta fuente se encuentran la combinación de un tratamiento a escala internacional con un enfoque sectorial que llega a un nivel considerable de detalle, así como la homogeneidad de los datos, todos ellos expresados en marcos alemanes. Los datos compilados proceden de diversas fuentes: de los anuarios de estadísticas agrarias de los principales Estados involucrados en el comercio de las materias primas; de los informes de asociaciones privadas de industriales textiles, principalmente británicas y alemanas, y de las estadísticas oficiales sobre comercio exterior de cada uno de los países. Los datos sobre la producción y el consumo fueron calculados a partir de las informaciones sobre producción y comercio de primeras materias, y exportaciones e importaciones de tejidos semi-elaborados y acabados. En estos cálculos se utilizaron coeficientes técnicos de transformación de las fibras en tejidos basados en los obtenidos para la industria textil alemana. Los precios de las distintas fibras fueron establecidos en función de su procedencia según las cotizaciones en los principales mercados alemanes. Los datos sobre comercio exterior proceden de las estadísticas oficiales de cada Estado y están sujetos a todos los problemas señalados en la bibliografía para este tipo de fuente ${ }^{2}$. A pesar de estas deficiencias que hay que tener en cuenta, se trata de una fuente de reconocida validez para el estudio del comercio internacional ${ }^{3}$.

Los datos utilizados en este trabajo han sido los correspondientes a las exportaciones de tejidos de algodón, tomando de forma agregada los tejidos semi-elaborados y los acabados. Para ello se han restado de los datos de exportación presentados por Kertesz, las informaciones acerca de las exportaciones de materia prima y de hilados. Cabe señalar que mientras los datos de exportación totales son exhaustivos para todos los países, en el caso de las desagregaciones por estado de elaboración solamente se hace referencia a las principales magnitudes. Esto podría introducir algún sesgo en los datos, en la medida en la que éstos podrían estar recogiendo algunos flujos de materias primas e hilos. Sin embargo, estos flujos deben ser necesariamente insignificantes, al no quedar recogidos en el apartado correspondiente a estos productos. Hay que señalar también que los datos sobre exportación se expresan en valores y se aplica para la conversión de monedas los tipos de cambio respecto al marco vigentes en $1913^{4}$.

\footnotetext{
2 Allen y Ely (1953), Morgenstern (1963).

${ }^{3}$ Federico y Tena (1991); Brown (1992) y (1995).

${ }^{4}$ Los tipos de cambio aplicados por Kertesz (1917) coinciden con los utilizados por
} 


\section{HIPÓTESIS SOBRE LA NATURALEZA DE LOS MERCADOS DE TEJIDOS DE ALGODÓN EN 1913}

Las hipótesis que se intentan contrastar están basadas en las nuevas teorías del comercio internacional y se sustentan en la evidencia empírica que pone de relieve un primer análisis descriptivo de los datos ${ }^{5}$. Bajo el supuesto general de imperfección de los mercados de tejidos de algodón, se proponen las siguientes hipótesis:

1. Histéresis histórica en los mercados de pasado colonial.

2. Especificidad del comercio europeo.

3. Diferenciación de producto.

Las relaciones coloniales, presentes o recientes en 1913, determinaban una parte importante de las exportaciones de tejidos de algodón, aunque el peso de los mercados coloniales variara sustancialmente entre Estados (Cuadro 1). Analizando el peso de la metrópoli sobre el mercado colonial se aprecian situaciones de considerable dominio económico, que ponen en duda la presencia de libre competencia en esos mercados, con la excepción de las colonias alemanas, que resulta de muy escasa relevancia cuantitativa (Cuadro 2).

\section{CUADRO 1}

El peso de los mercados coloniales sobre la exportación (porcentajes)

\begin{tabular}{|c|c|}
\hline & Mercados coloniales ** \\
\hline 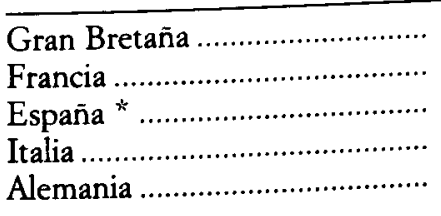 & $\begin{array}{r}33,01 \\
38,64 \\
41,97 \\
5,05 \\
0,47 \\
\end{array}$ \\
\hline 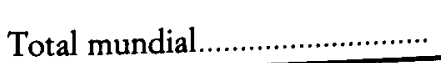 & 31,25 \\
\hline
\end{tabular}

Notas: * Se incluyen Cuba, Puerto Rico y Filipinas.

** Se consideran las colonias presentes, pero también las ex colonias.

FUENTE: Kertesz (1917).

Maizels (1963) para el mismo período histórico, siendo, los primeros, respecto al marco, y los segundos, respecto al dólar.

5 Carreras (1998). 


\section{CUADRO 2}

El peso de las metrópolis sobre los mercados coloniales

\begin{tabular}{|c|c|}
\hline & $\begin{array}{c}\text { Porcentaje del mercado } \\
\text { colonial }\end{array}$ \\
\hline Gran Bretaña & 92,71 \\
\hline 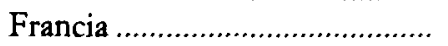 & 67,14 \\
\hline 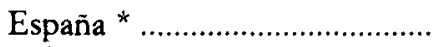 & 65,49 \\
\hline 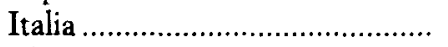 & 69,44 \\
\hline 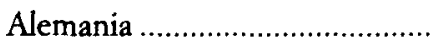 & 8,75 \\
\hline
\end{tabular}

Notas: * Se incluyen Cuba, Puerto Rico y Filipinas.

** Se consideran las colonias presentes, pero también las ex colonias.

FUENTE: Kertesz (1917).

\section{CUADRO 3}

El peso del comercio intraeuropeo (porcentajes)

\begin{tabular}{|c|c|}
\hline & Mercados europeos \\
\hline 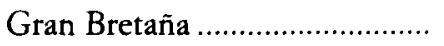 & 18,20 \\
\hline 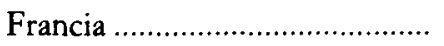 & 28,22 \\
\hline 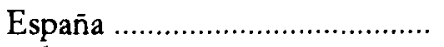 & 4,38 \\
\hline 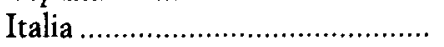 & 17,44 \\
\hline 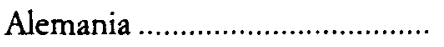 & 53,95 \\
\hline 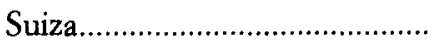 & 53,76 \\
\hline 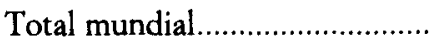 & 30,70 \\
\hline
\end{tabular}

FUENTE: Kertesz (1917).

El análisis geográfico de las exportaciones de tejidos de algodón muestra la configuración de un área comercial centrada en Europa occidental (Cuadro 3). En este mercado intraeuropeo se observan características específicas, lo que podría deberse a una mayor presencia de intercambios comerciales de tipo intraindustrial.

La diferenciación de producto, entendida tanto como diferenciación por calidades como adaptación a preferencias específicas de determinados segmentos de mercado, parece ser una estrategia comercial fundamental en las exportaciones de tejidos de algodón de 1913. Esta hipótesis surge a partir de algunos estudios sobre el sector textil que han mostrado la de- 
bilidad de las explicaciones basadas exclusivamente en las dotaciones factoriales. Asimismo, estudios precedentes sobre diferenciación de producto e informes comerciales de la época destacan la importancia del tipo de producto para la competitividad en la exportación de tejidos de algodón ${ }^{6}$.

Hay que señalar que el término diferenciación de producto carece de un significado unívoco al aplicarse a los mercados de tejidos de algodón, ya que puede referirse a diferencias en las calidades de los tejidos (ya sea por los distintos hilos utilizados o por el número de hilos incorporados al tejido), a diferencias en los acabados (distintos colores, tintes, diseños, estampados, ...); y a diferencias en los estados de elaboración (tejidos crudos, semi-elaborados o acabados totalmente). Estas tres acepciones del término diferenciación pueden suponer cosas muy distintas. Las diferencias en las calidades se pueden relacionar con limitaciones técnicas y niveles de renta de la demanda; las diferencias en los acabados pueden relacionarse con las preferencias de los consumidores y, por tanto, con la importancia de las redes de información desde los mercados hacia los comerciantes y fabricantes y, finalmente, las diferencias en los estados de elaboración se pueden relacionar con procesos de especialización productiva horizontal que superarian las fronteras estatales para pasar a conformar regiones económicas supranacionales. La complejidad de la diferenciación de producto y de los procesos de especialización productiva horizontal no ha sido abordada en el análisis desarrollado a continuación, sino que simplemente se ha calculado un indice de comercio intraindustrial como primera aproximación. Esta simplificación debe tenerse en cuenta en la interpretación de los resultados.

\section{ESPECIFICACIÓN DE LA ECUACIÓN GRAVTTACIONAL PARA EL COMERCIO TEXTIL BILATERAL}

La elección de un modelo de tipo gravitacional para el análisis de los mercados internacionales de tejidos de algodón se justifica como alternativa a los modelos de dotación de factores aplicados al comercio del período anterior a la Primera Guerra Mundial, así como a las explicaciones acerca del fracaso exportador de la industria algodonera española centradas exclu-

' Clark (1987); Sudrià (1983) y (1999); Sandberg (1968); Temin (1988); Brown (1992) y (1995); Feenstra, Markusen y Rose (1998); Graham Clark (1908a) y (1908b); Report of the Tariff Board (1912). 
sivamente en los costes de los factores ${ }^{7}$. Esta opción metodológica se fundamenta en los análisis con supuestos de competencia imperfecta desarrollados por algunos autores en el ámbito internacional, así como en la bibliografía española que destaca como causas del fracaso exportador las variables de demanda interna, economías de escala (tanto en la producción como en la comercialización), la pérdida de las colonias o los condicionantes geográficos ${ }^{8}$. La ecuación gravitacional especificada para contrastar las hipótesis planteadas se basa en la utilizada por Feenstra, Markusen y Rose, bajo la siguiente expresión ${ }^{9}$ :

EXPORT $_{i j}=\mathrm{C}(1)+\mathrm{C}(2)^{*} \mathrm{PIBX}_{\mathrm{i}}+\mathrm{C}(3)^{*} \mathrm{PIBM}_{\mathrm{j}}+\mathrm{C}(4)^{*} \mathrm{CENTR}_{\mathrm{i}}+$ $+\mathrm{C}(5)^{\star} \mathrm{DOMCOL}_{\mathrm{ij}}+\mathrm{C}(6)^{\star} \mathrm{INTRA}_{\mathrm{ij}}$

donde: EXPORT $_{\mathrm{ij}}$ es la variable endógena, expresada como el logaritmo de los intercambios bilaterales de tejidos de algodón según aparecen en Kertesz ${ }^{10}$. Esta variable incluye los flujos comerciales de 18 Estados exportadores con sus respectivos mercados importadores. Cada dato recoge el intercambio de tejidos de algodón entre un Estado exportador y uno de sus importadores, de modo que los datos se repiten para cada uno de los socios comerciales de cada Estado exportador ${ }^{11}$.

PIBX $_{i}$ es el PIB del Estado exportador que recoge el peso del tamaño del mercado interior sobre las exportaciones (home market effect) ${ }^{12}$. En esta variable se recogen dos fenómenos distintos relacionados con la influencia del mercado interior. En primer lugar, la incidencia de posibles economías de escala debidas a un tamaño de mercado grande que, según las teorías del desbordamiento, fomentarían la exportación por la vía del abaratamiento de los costes de producción. En segundo lugar, la importancia de mercados de renta elevada que justificaría especializaciones a través de la diversificación de producto.

$\mathrm{PIBM}_{\mathrm{j}}$ es el PIB del Estado importador que recoge el peso del tamaño del mercado importador sobre las exportaciones, es decir, el «efecto atracción» de la demanda exterior. Según Feenstra, Markusen y Rose, si el coeficiente de $\mathrm{PIBX}_{\mathrm{i}}$ excede al de $\mathrm{PIBM}_{\mathrm{j}}$ se puede presuponer que el

${ }^{7}$ Estevadeordal (1993).

* Nadal (1975) y (1992), Nadal y Sudrià (1993), Sudrià (1999).

${ }^{9}$ Feenstra, Markusen y Rose (1998).

10 Kertesz (1917).

1 Las observaciones disponibles para esta variable (302) se han visto reducidas debido a la escasez de datos en las variables exógenas, dejando el tamaño de la muestra en 225 observaciones.

12 Los datos sobre el PIB proceden de Maddison (1995). 
efecto desbordamiento supera al efecto de atracción, es decir, estaríamos ante la presencia de home market effect ${ }^{13}$.

$\mathrm{CENTR}_{\mathbf{i}}$ pretende capturar la influencia de una localización estratégica en relación a los mercados consumidores de tejidos de algodón ponderando la distancia geográfica por factores económicos ${ }^{14}$. Para ello, se expresa del siguiente modo:

$$
\mathrm{CENTR}_{\mathrm{i}}=\Sigma\left[\mathrm{PIB}_{\mathrm{j}} / \mathrm{DIST}_{\mathrm{ij}}\right]+\mathrm{PIB}_{\mathrm{i}}
$$

donde: PIBj es el PIB del mercado importador; DIST $_{i j}$ es la distancia lineal entre la capital del Estado exportador y la del importador; y PIB $_{i}$ es el PIB del mercado interior. De este modo, esta variable aumenta o bien a causa de la proximidad a los mercados consumidores (por ejemplo, en el caso de Alemania o Suiza respecto a los mercados europeos), o bien por el gran tamaño de los mercados aun siendo éstos distantes geográficamente (por ejemplo, en el caso de Gran Bretaña respecto a la India).

$\mathrm{DOMCOL}_{\mathrm{ij}}$ es la variable utilizada para la histéresis histórica derivada de dependencias políticas, expresada como una variable dummy que toma el valor 1 ante la presencia de una relación colonial entre ambos socios comerciales, presente o reciente en 1913; y 0 en el resto de los casos ${ }^{15}$. Una manera alternativa de recoger procesos de histéresis histórica o path dependence sería la inclusión de los flujos comerciales del pasado, bajo el supuesto de que las exportaciones del pasado favorecen las exportaciones del presente, debido, por ejemplo, al establecimiento de economías de escala en la comercialización, a su vez debidas a las redes de información establecidas en el pasado. Esta variable evitaría los problemas de interpretación que puede suscitar la variable del dominio colonial. Sin embargo, no se han utilizado los datos sobre flujos comerciales bilaterales tanto por la dificultad para contar con estos datos para períodos anteriores a 1913, como por las dificultades econométricas que una variable retardada introduce en el modelo. Por todo ello, hay que insistir en que la variable escogida no pretende captar la influencia de los mercados coloniales, per se, sino como aproximación a los procesos de histéresis histórica, debidos, quizás, al establecimiento de economías de escala en la comercialización. En este

${ }^{13}$ Feenstra, Markusen y Rose (1998).

14 Otra aproximación a la distancia geográfica usada por algunos autores sería una variable dummy de frontera común. Esta variable, sin embargo, ha sido descartada debido a su significado ambiguo, al combinar el efecto de la proximidad geográfica con el de la presencia de barreras arancelarias, efectos ambos que, al ser de signo opuesto, tienden a cancelarse entre sí.

${ }^{15}$ Eichengreen e Irwin (1996). 
sentido, resulta paradigmático el caso español, en el que un porcentaje importante de las exportaciones en 1913 se dirigen a las ex-colonias, no por el mantenimiento del dominio político, sino, probablemente, por la persistencia de los canales de comercialización. Hay que señalar, por último, que los procesos de histéresis histórica pueden producirse por otras causas, por ejemplo, los flujos migratorios, que tendrian estrecha relación con la variable de dominio colonial.

INTRA $_{\mathrm{ij}}$ pretende aproximar el efecto de la diferenciación de producto, a través de los flujos de comercio intraindustrial. Para ello, se utiliza el índice Grubel-Lloyd expresado como:

$$
\operatorname{INTRA}_{\mathrm{ij}}=\left[\min \left(\mathrm{X}_{\mathrm{ij}}, \mathrm{X}_{\mathrm{ji}}\right)\right] /\left[\mathrm{X}_{\mathrm{ij}}+\mathrm{X}_{\mathrm{ji}}\right]
$$

donde $X_{i j}$ es la exportación entre el Estado i (exportador) y el $j$ (importador), mientras que $\mathrm{X}_{\mathrm{ji}}$ recoge la exportación en sentido inverso. Se trata de una simplificación, seguramente excesiva, del fenómeno de la diferenciación de producto, pero que sirve, en todo caso, para evaluar el alcance del comercio intraindustrial en los mercados internacionales.

\section{ESTIMACIÓN Y ANÁLISIS DE SENSIBILIDAD DE LA ECUACIÓN GRAVITACIONAL}

La estimación se ha corregido por la posible heterocedasticidad y se ha realizado para una muestra de 225 observaciones que comprenden a los principales Estados exportadores de tejidos de algodón y a sus socios comerciales ${ }^{16}$. Los resultados aparecen en el Cuadro 4. En la columna 1, todas las variables exógenas se muestran como significativas. La histéresis histórica (DOMCOL) es, sin embargo, la variable que alcanza mayor significación. Si se comparan los coeficientes de las variables de renta, se observa que la dependencia de las exportaciones respecto al mercado interior supera ligeramente a la de la demanda externa. Esto podría indicar la presencia del bome market effect, siguiendo la interpretación de Feenstra, Markusen y Rose ya mencionada. Sin embargo, la significación de esta diferencia de coeficientes no es demasiado grande con lo que parece que el papel de la demanda interior y el de la demanda exterior tendrían efectos parecidos en el fomento de las exportaciones. Por otro lado, la significación

${ }^{16}$ Alemania, Austria-Hungría, Bélgica, Dinamarca, Estados Unidos, España, Finlandia, Francia, Gran Bretaña, Holanda, India Británica, Italia, Japón, Rusia, Suecia, Suiza, Turquía y China. 


\section{CUADRO 4}

Estimación de la ecuación gravitacional

\begin{tabular}{|c|c|c|c|c|}
\hline \multirow{2}{*}{ Variables independientes } & \multirow{2}{*}{$\frac{\text { Estimación }}{(1)}$} & \multicolumn{3}{|c|}{ Análisis de sensibilidad } \\
\hline & & (2) & (3) & (4) \\
\hline C..... & $\begin{array}{l}-0,989 \\
(-4,850)\end{array}$ & $\begin{array}{l}-0,969 \\
(-4,787)\end{array}$ & $\begin{array}{l}-0,625 \\
(-2,768)\end{array}$ & $\begin{array}{c}-1,054 \\
(-3,788)\end{array}$ \\
\hline PIBX.. & $\begin{array}{c}0,456 \\
(5,472)\end{array}$ & - & $\begin{array}{c}0,248 \\
(2,666)\end{array}$ & $\begin{array}{c}0,560 \\
(5,276)\end{array}$ \\
\hline PIBM.... & $\begin{array}{c}0,334 \\
(4,517)\end{array}$ & - & $\begin{array}{c}0,328 \\
(4,276)\end{array}$ & $\begin{array}{c}0,204 \\
(1,850)\end{array}$ \\
\hline CENTR. & $\begin{array}{l}1,040 \\
(3,630)\end{array}$ & $\begin{array}{c}0,893 \\
(2,418)\end{array}$ & $\begin{array}{c}0,548 \\
(1,927)\end{array}$ & $\begin{array}{r}0,706 \\
(1,422)\end{array}$ \\
\hline DOMCOL & $\begin{array}{l}1,090 \\
(7,658)\end{array}$ & $\begin{array}{l}1,080 \\
(7,791)\end{array}$ & $\begin{array}{l}0,918 \\
(3,644)\end{array}$ & - \\
\hline INTRA.. & $\begin{array}{c}0,371 \\
(2,340)\end{array}$ & $\begin{array}{c}0,426 \\
(2,599)\end{array}$ & $\begin{array}{c}0,388 \\
(2,193)\end{array}$ & $\begin{array}{c}0,693 \\
(3,663)\end{array}$ \\
\hline 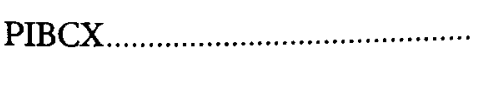 & - & $\begin{array}{c}0,594 \\
(2,804)\end{array}$ & - & - \\
\hline (n) & - & $\begin{array}{c}0,203 \\
(1,386)\end{array}$ & - & - \\
\hline POBX. & - & $\begin{array}{c}0,415 \\
(4,776)\end{array}$ & - & - \\
\hline POBM & - & $\begin{array}{c}0,343 \\
(4,583)\end{array}$ & - & - \\
\hline 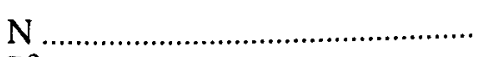 & 225 & 225 & 190 & 100 \\
\hline $\mathrm{R}^{2}$ & $\begin{array}{l}0,310 \\
0,294\end{array}$ & $\begin{array}{l}0,316 \\
0,294\end{array}$ & $\begin{array}{l}0,206 \\
0,184\end{array}$ & $\begin{array}{l}0,338 \\
0,310\end{array}$ \\
\hline
\end{tabular}

NOTAS: (1) Estimación para la muestra completa; (2) Análisis de sensibilidad alterando el número de variables explicativas; (3) Análisis de sensibilidad excluyendo el caso británico; (4) Análisis de sensibilidad incluyendo sólo el comercio intraeuropeo. Para la definición de las variables, véase el texto.

de las variables de renta podría ser indicativa del peso de las estrategias de competitividad vía calidad o diferenciación del producto, mientras que la significación de la variable histórica indicaría más bien la presencia de economías de escala en la comercialización.

En términos agregados, el modelo alcanza niveles de significación aceptables. Se han realizado una serie de tests para verificar su grado de sensibilidad. El primero ha consistido en alterar la cantidad de variables exógenas (Cuadro 4, columna 2). Para ello, se han descompuesto las variables 
de renta en dos nuevas variables: la renta per cápita (PIBC) y la población (POB). El aumento de las variables exógenas no parece alterar significativamente los resultados. La descomposición de las variables de renta se traduce en una mayor significación de la población en relación con los ingresos per cápita, tanto para el mercado interior como para el mercado exterior, lo que indicaría mayor importancia de las economías de escala que de las preferencias por la diversificación o la calidad. La comparación de los coeficientes de las nuevas variables de renta muestra una mayor distancia entre el efecto del mercado interior y el del mercado exterior, lo que podría ser interpretado como un mayor peso del bome market effect en términos per cápita, es decir, que la elasticidad renta de la exportación respecto al mercado interior superaría la elasticidad renta respecto al mercado exterior.

Un segundo análisis de sensibilidad ha consistido en fragmentar la muestra sobre la que se ha realizado la estimación considerando la gran desigualdad en la distribución de las exportaciones de tejidos de algodón entre Estados (Cuadro 5). Teniendo en cuenta que Gran Bretaña concentra el 57 por 100 de la exportación, se podría considerar que su inclusión en la muestra podría estar afectando la estimación, pues el patrón británico de exportación pudiera estar superponiéndose al del resto de países. De hecho, el caso británico ha sido considerado a menudo por la literatura como un caso «atípico». Sin embargo, su exclusión no parece alterar significativamente los resultados (Cuadro 4, columna 3).

El tercer análisis de sensibilidad se ha realizado considerando la hipótesis de que el comercio intraeuropeo pudiera seguir un comportamiento diferenciado del resto del comercio mundial ${ }^{17}$. En este caso, se han reducido tanto la cantidad de variables exógenas como el número de observaciones (Cuadro 4, columna 4). La variable histórica de la dependencia colonial ha sido suprimida, ya que su valor era nulo para todos los intercambios intraeuropeos. En este tercer análisis de sensibilidad, la significación de las variables se altera, especialmente en el caso de la variable geográfica y de la renta del mercado importador ${ }^{18}$. Esto podría indicar que, para el caso de los intercambios intraeuropeos, las variables explicativas

17 Incluyendo Gran Bretaña en sus relaciones comerciales con la Europa continental.

${ }_{18}$ En el caso del comercio intraeuropeo, seguramente tendría más sentido especificar la variable geográfica como contigüidad de fronteras, más que en función de la distancia entre capitales. Resulta un tanto sorprendente que la variable que recoge el peso de la demanda externa no resulte significativa para el caso intraeuropeo, cuando es de suponer que en este caso la demanda debería influir en la exportación. Sin embargo, debe tenerse en cuenta que la relativa homogeneidad en la renta de estos mercados puede ser la causa 


\section{CUADRO 5}

Distribución de la Exportación Mundial

\begin{tabular}{|c|c|c|}
\hline Exportadores & $\begin{array}{l}\text { Millones } \\
\text { de marcos }\end{array}$ & Porcentajes \\
\hline Gran Bretaña ........................ & 1.989 .538 & 57,06 \\
\hline 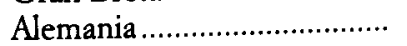 & 491.812 & 14,10 \\
\hline 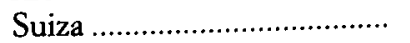 & 180.541 & 5,17 \\
\hline 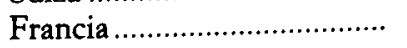 & 166.387 & 4,77 \\
\hline Italia ...................... & 126.720 & 3,63 \\
\hline EEUU & 115.936 & 3,32 \\
\hline 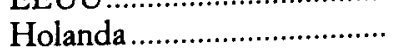 & 114.175 & 3,27 \\
\hline Austria-Hungría .................... & 113.235 & 3,24 \\
\hline 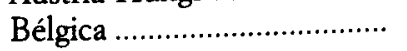 & 64.681 & 1,85 \\
\hline 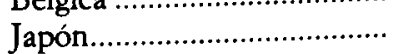 & 58.679 & 1,68 \\
\hline 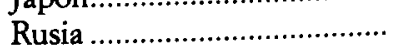 & 31.500 & 0,90 \\
\hline 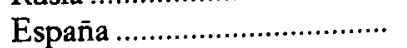 & 18.100 & 0,51 \\
\hline 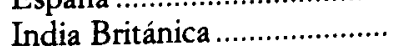 & 6.403 & 0,18 \\
\hline 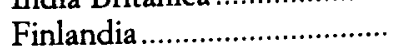 & 3.800 & 0,10 \\
\hline 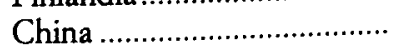 & 3.116 & 0,08 \\
\hline 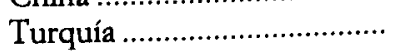 & 0,889 & 0,02 \\
\hline 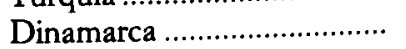 & 0,600 & 0,01 \\
\hline 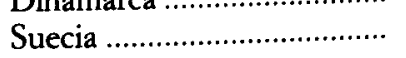 & 0,500 & 0,01 \\
\hline ............... & 3.486 .612 & 100 \\
\hline
\end{tabular}

FUENTE: Kertesz (1917).

relevantes deberían basarse en la presencia del bome market effect y del comercio intraindustrial, y reforzaría así la segunda de las hipótesis planteadas.

\section{CONCLUSIONES}

Para volver al objetivo principal de este artículo es preciso retomar el debate historiográfico acerca de la escasa relevancia exportadora de la

de esta baja significación. Para este caso, tendría más sentido especificar la variable renta como la diferencia entre la renta del mercado interior y la del mercado importador (PIBX-PIBM), considerando que una escasa distancia entre ambos niveles de renta fomentaría la exportación. 
industria algodonera española. La pretensión del trabajo no consistía en una demostración empírica de las causas determinantes de este fenómeno, sino en la propuesta de un enfoque distinto que puede generar nuevas aportaciones al debate. Para ello, se ha trasladado el problema al contexto internacional, gracias a una fuente estadística relativamente inédita y adoptando un marco teórico basado principalmente en la presunción de imperfección de los mercados. Asimismo, se ha aplicado una metodología que, aunque parcial, permite incluir nuevas variables explicativas.

El supuesto de imperfección de los mercados internacionales de tejidos de algodón se ha concretado en tres hipótesis. La primera contemplaba el peso de las dependencias históricas de los pasados coloniales en la determinación de los flujos comerciales. El análisis meramente descriptivo de los datos ya proporcionaba una primera idea de la importancia de los mercados coloniales en la exportación de tejidos de algodón. El contraste econométrico ha confirmado esta hipótesis, lo que pone de relieve la importancia de los procesos de histéresis histórica. Esta parte del mercado debería explicarse más mediante un análisis histórico que mediante un análisis estrictamente económico, orientándose hacia las llamadas teorías de la path dependence. Sin embargo, cabe plantearse las implicaciones de estos procesos en la competitividad de las metrópolis en el largo plazo. Si bien se ha realizado un análisis sincrónico, estableciendo que en el año 1913 las relaciones coloniales eran un factor relevante para las exportaciones de tejidos de algodón, cabría preguntarse hasta cuándo pueden mantenerse estas posiciones privilegiadas sobre los mercados, es decir, cómo evolucionan en el tiempo los procesos de histéresis histórica capaces de crear ventajas competitivas en sí mismos. Para ello, habría que analizar las redes de información y comercialización, lo que escapa de las posibilidades del modelo planteado en este trabajo.

La segunda hipótesis se centraba en el análisis de los mercados europeos y planteaba la presencia de pautas comerciales basadas en un mayor peso de la demanda y en la presencia de flujos comerciales de tipo intraindustrial. El análisis de los datos revelaba que Europa suponía un porcentaje muy importante de los mercados de los principales exportadores de tejidos de algodón, incluida Gran Bretaña. La estimación econométrica muestra niveles aceptables de significación tanto para las variables de demanda como para el índice de comercio intraindustrial; mientras que en la restricción de la muestra a los flujos intraeuropeos tan sólo estas dos variables mantenían su significación. Del análisis realizado se puede sugerir que la capacidad de exportación a Europa podría estar determinada por estrategias 
alternativas a la competencia vía precios, y que éstas también podrían resultar beneficiosas para la exportación al resto de los mercados. Este tipo de estrategias dependerían en gran medida de las características de los mercados interiores, no tanto por su tamaño en términos absolutos, sino por su capacidad adquisitiva y las características de sus preferencias.

La tercera hipótesis se basaba en el supuesto de la no homogeneidad de los tejidos de algodón. Este supuesto partía de informaciones cualitativas recogidas en informes comerciales de la época así como de trabajos empíricos anteriores, que han estimado la presencia de esta diferenciación de producto. Ya se ha advertido que esta hipótesis entraña mayor complejidad de la que contempla el modelo gravitacional, ya que puede referirse tanto a diferenciación por calidades como a diferenciación por acabados o por estado de elaboración, implicando ésta procesos de especialización productiva horizontal. La aproximación utilizada en este trabajo ha sido el indice de comercio intraindustrial de Grubel-Lloyd, que aparece significativo. Cabría profundizar más en esta variable intentando establecer tipologías de producto e intentando distinguir entre las diferentes estrategias de diferenciación de producto y los procesos de especialización productiva horizontal. La importancia de las primeras podría aproximarse también a través de la significación de la variable renta per cápita del mercado interior incluida en el primer análisis de sensibilidad.

Para finalizar, habría que ver cómo se trasladan estas hipótesis al caso español. Si los mercados coloniales resultan relevantes como generadores de procesos de histéresis histórica, tanto la escasez de colonias propias como un acceso tardío a los mercados «libres», pueden tomarse como obstáculos a la exportación. Asimismo, se pone de relieve la necesidad de explicar la ausencia española en los mercados europeos, sobre todo comparada con el caso italiano: mientras que los mercados europeos representan el 4 por 100 de la exportación española, ese porcentaje alcanza el 17 por 100 en el caso italiano. La diferencia parece significativa y merece ser tenida en cuenta en el desarrollo futuro de la investigación.

En el presente análisis no se han tenido en cuenta las diferencias de costes de producción, por lo que la poca capacidad exportadora de la industria algodonera española podría resultar de la ineficiencia de las estrategias de diferenciación de producto en las exportaciones; esto no significa que los costes productivos también puedan desempeñar un papel determinante ${ }^{19}$. Quedaría por ver en qué medida ello se debe a factores de

${ }^{19}$ Crafts (1989), Crafts y Thomas (1986), Estevadeordal (1993), Rosés (1998a) y (1998b). 
demanda, es decir, a diferencias de renta y de preferencias entre el mercado español y los mercados europeos, a factores técnicos o a problemas derivados de la comercialización, como la ausencia de redes de información. Para ello parece necesario profundizar más en los procesos de comercialización internacional de tejidos de algodón.

\section{BIBLIOGRAFÍA}

Allen, R. G. D., y Ely, E. J. (1953): International Trade Statistics, New York, John Wiley \& Sons.

BrowN, J. C. (1992): «Market organization, Protection, and Vertical Integration: German Cotton Textiles before 1914», The Joumal of Economic History, 52, 2, pp. 339-351.

- (1995): «Imperfect Competition and Anglo-German Trade Rivalry: Markets for Cotton Textiles before 1914», The Journal of Economic History, 55, 3, pp. 494-527.

CARRERAS, A. (1998): «El comerç tèxtil mundial el 1913: lliure competència o colonialisme», Trabajo de investigación del Doctorado en Historia Económica del Departamento de Historia e Instituciones Económicas de la Universidad de Barcelona, dirigido por C. Sudrià.

ClARK, G. (1987): "Why isn't the Whole World Developed? Lessons From the Cotton Mills», Joumal of Economic History , 47, 1, pp. 141-173.

CrafTS, N. F. R. (1989): «Revealed Comparative Advantage in Manufacturing, 1899-1950», The Joumal of European Economic History, 18, 1, pp. 127-137.

Crafts, N. F. R. y Thomas, M. (1986): «Comparative Advantage in UK Manufacturing Trade, 1910-1935», The Economic Journal, 96, pp. 629-645.

EICHEngreen, B., e IrWIN, D. A. (1996): «The Role of History in Bilateral Trade Flows», National Bureau of Economic Research, Working Paper 5565.

EstevadeORDAL, A. (1993): «Historical Essays on Comparative Advantage: 1913-1938», Tesis Doctoral, Harvard University.

Federico, G., y TenA, A. (1991): «On the Accurancy of Foreign Trade Statistics (1909-1935): Morgenstern Revisited», Explorations in Economic History, 28, 3, pp. 259-273.

Feenstra, R. C.; Markusen, J. A., y Rose, A. K. (1998): «Understanding the Home Market Effect and the Gravity Equation: The Role of Differentiating Goods», National Bureau of Economic Research, Working Paper 6804.

Graham Clark, W. A. (1908a): «Cotton Fabrics in Middle Europe: Germany, Austria-Hungary, Switzerland», Department of Commerce and Labor Bureau of Manufactures, Washington.

- (1908b): «Cotton Textile Trade in Turkish Empire, Greece and Italy», Department of Commerce and Labor Bureau of Manufactures, Washington.

KertesZ, A. (1917): Die Textilindustrie sämtlicher Staaten. Entwicklung, Erzeugung, Absatzverbältnisse. Nach den statistischen Unterlagen der einzelnen Staaten für die Baumwoll-, Woll-, Seiden-, Leine-, Jute- und Konfektions-Industrie als zweite 
Auflage der «Textilindustrie Deutschlands im Welthandel, Braunschweig Viewig \& Sohn.

MADDISON, A. (1995): Monitoring the world economy 1820-1992, Paris, OECD. MAtzels, A. (1963): Industrial Growth and World Trade. An empirical study of trends in production, consumption and trade in manufactures from 1899-1959 with a discussion of probable future trends, London, Cambridge University Press

Morgernstern, O. (1963): «Foreign Trade Statistics», en On the Accuracy of Economic Observations, New Jersey, Princeton University Press, pp. 137-180.

NADAL, J. (1975): El fracaso de la revolución industrial en España, 1814-1913, Barcelona, Ariel.

- (1992): Moler, tejer y fundir. Estudios de historia industrial, Barcelona, Ariel.

NADAL, J., y SuDRiA, C. (1993): «La controversia en torno al atraso económico español en la segunda mitad del siglo XIX», Revista de Historia Industrial, 4, pp. 199-227.

RePORT Of THE TaRiff Board on Shedule I of THE TARIFF Law (1912): Cotton Manufacturers, House of Representatives, vol. 1, Washington.

Rosés, J. R. (1998a): «The Early Phase of Catalan Industrialisation, 1830-1861», Tesis Doctoral, European University Institute, Florencia.

- (1998b): «The Choice of Technology in the Mediterranean Basin: Some Evidence From the Spanish, Italian, British and US Cotton Mills (1830-1860)», Universidad Carlos III, Working Papers, pp. 98-99.

SANDBERG, L. G. (1968): «Movements in the Quality of British Cotton Textile Exports, 1815-1913», The Journal of Economic History, 28, 1, pp. 1-27.

SUDRIÄ, C. (1983): «La exportación en el desarrollo de la industria algodonera española, 1875-1920», Revista de Historia Económica, 1, 2, pp. 369-386.

- (1999): «La empresa española y los problemas de competitividad internacional. Una visión desde la industria algodonera» (manuscrito).

Temin, P. (1988): «Product Quality and Vertical Integration in the Early Cotton Textile Industry», The Journal of Economic History, 48, 4, pp. 891-907. 